\title{
Self-rated mental stress and exercise training response in healthy subjects
}

\author{
Piritta S. Ruuska ${ }^{1}$, Arto J. Hautala ${ }^{1}$, Antti M. Kiviniemi ${ }^{1}$, Timo H. Mäkikallio ${ }^{2}$ and Mikko P. Tulppo ${ }^{1,2} *$ \\ 1 Department of Exercise and Medical Physiology, Verve, Oulu, Finland \\ ${ }^{2}$ Department of Internal Medicine, Institute of Clinical Medicine, University of Oulu, Finland
}

\section{Edited by:}

Kari Kalliokoski, University of Turku Finland

Reviewed by:

Chia-Hua Kuo, University of Texas at Austin, USA

Anthony Leicht, James Cook

University, Australia

Celena Scheede-Bergdahl, McGill

University, Canada

${ }^{*}$ Correspondence:

Mikko P. Tulppo, Department of

Exercise and Medical Physiology,

Verve, P.O. Box 404, Fl-90101 Oulu,

Finland.

e-mail:mikko.tulppo@verve.fi

\begin{abstract}
Purpose: Individual responses to aerobic training vary from almost none to a $40 \%$ increase in aerobic fitness in healthy subjects. We hypothesized that the baseline self-rated mental stress may influence to the training response. Methods: The study population included 44 healthy sedentary subjects ( 22 women) and 14 controls. The laboratory controlled training period was 2 weeks, including five sessions a week at an intensity of $75 \%$ of the maximum heart rate for $40 \mathrm{~min} / \mathrm{session}$. Self-rated mental stress was assessed by inquiry prior to the training period from 1 (low psychological resources and a lot of stressors in my life) to 10 (high psychological resources and no stressors in my life), respectively. Results: Mean peak oxygen uptake $\left(V_{\mathrm{O}_{2} \text { peak }}\right)$ increased from $34 \pm 7$ to $37 \pm 7 \mathrm{ml} \mathrm{kg}^{-1} \mathrm{~min}^{-1}$ in training group ( $p<0.001$ ) and did not change in control group (from $34 \pm 7$ to $34 \pm 7 \mathrm{ml} \mathrm{kg}^{-1} \mathrm{~min}^{-1}$ ). Among the training group, the self-rated stress at the baseline condition correlated with the change in fitness after training intervention, e.g., with the change in maximal power $(r=0.45, p=0.002, \mathrm{~W} / \mathrm{kg})$ and with the change in $V_{\mathrm{O}_{2} \text { peak }}(r=0.32, p=0.039$, $\mathrm{ml} \mathrm{kg}^{-1} \mathrm{~min}^{-1}$ ). The self-rated stress at the baseline correlated with the change in fitness in both female and male, e.g., $r=0.44, p=0.039$ and $r=0.43, p=0.045$ for $\Delta \mathrm{W} / \mathrm{kg}$ in female and male, respectively. Conclusion: As a novel finding the baseline self-rated mental stress is associated with the individual training response among healthy females and males after highly controlled aerobic training intervention. The changes in fitness were very low or absent in the subjects who experience their psychological resources low and a lot of stressors in their life at the beginning of aerobic training intervention.
\end{abstract}

Keywords: mental stress, physical fitness, aerobic exercise, sex differences

\section{INTRODUCTION}

Regular physical activity and good physical fitness are widely accepted as factors that reduce all-cause mortality and improve a number of health outcomes (Kesäniemi et al., 2001; Kurl et al., 2003; Laukkanen et al., 2004; LaMonte et al., 2005). A study of Myers et al. (2002) showed that low maximal aerobic capacity is closely related to an increase of untoward cardiac events. Therefore, physical training has been proposed to reduce these events by improving aerobic capacity (Billman, 2002). However, enormous heterogeneity in the responsiveness to physical training assessed as the change in peak oxygen uptake $\left(V_{\mathrm{O}_{2} \text { peak }}\right)$, has been observed even in highly standardized training programs (Bouchard and Rankinen, 2001; Hautala et al., 2003, 2006). The mean improvements to $\left(V_{\mathrm{O}_{2} \text { peak }}\right)$ have been about $25 \%$ of the baseline values, but with a range from no gain to a $40 \%$ increase in $V_{\mathrm{O}_{2} \text { peak }}$ (Bouchard, 1995; Bouchard and Rankinen, 2001). Genetic factors may explain a major part ( $\sim 50 \%)$ of the inter-individual variation in training response (Bouchard et al., 1999, 2011; Hautala et al., 2007; Timmons et al., 2010; Hagberg et al., 2011). Furthermore, sex, race, and baseline fitness level together accounted for $11 \%$ of the variance in the response to 20 weeks of standardized training (Bouchard and Rankinen, 2001).

In our previous studies heart rate (HR) variability has been associated with training response accounting for $27 \%$ of the change as an independent predictor of the aerobic training response (Hautala et al., 2003; Tulppo et al., 2003). Particularly, low beat-to-beat HR variability, an index of cardiac vagal tone, has shown to be associated with poor training response (Hautala et al., 2003). The magnitude of HR variability is a result of complex interaction between the peripheral and central autonomic regulation mechanisms (Tulppo et al., 2005) favoring the hypothesis that also the central nervous system state may be involved in the training adaptation.

In the previous cross sectional study, a good self-rated mental health was positively associated with aerobic fitness in a large population of healthy subjects (Galper et al., 2006). Also a large body of studies shows that physical training improves mental health, e.g., by reducing symptom of depression (Dunn et al., 2001). Furthermore, dehydroepiandrosterone sulfate (DHEA-S) has shown to be associated with individual differences in exercise training responses (Huang et al., 2006), adaptation to the high altitude conditions (Lee et al., 2006), and the coping mechanism against psychological challenge (Wang et al., 2009). DHEA-S has shown to buffer stress and playing a role in functional recovery in humans. This is emphasizing the potential role of stress in training adaptation (Regelson et al., 1988; Herbert, 1998; Cruess et al., 1999). However, the association between baseline mental stress and training adaptation is largely unknown. Therefore, the 
purpose of this study was to test the hypothesis that individual selfrated psychological resources/stress may predict the response to highly controlled aerobic training in healthy subjects. We assessed self-rated psychological resources/stress among subjects who subsequently underwent a highly controlled aerobic exercise training intervention at a laboratory condition.

\section{MATERIALS AND METHODS SUBJECTS}

The subjects were recruited by newspaper ads. All smokers, subjects with body mass index $(\mathrm{BMI})>32 \mathrm{~kg} / \mathrm{m}^{2}$, subjects who did regular physical training more than two times per week, and subjects with diabetes mellitus, asthma, any medications or cardiovascular disorders were excluded. We invited 62 subjects to our laboratory (Dept. of Exercise and Medical Physiology, Verve, Oulu, Finland) for a more specific assessment. The number of subjects was selected on the basis of a priori power analysis to give $90 \%$ power to the responsiveness of $V_{\mathrm{O}_{2} \text { peak }}$ after 2 weeks of endurance training. According the previous study (Hautala et al., 2003), 8\% improvement in $V_{\mathrm{O}_{2} \text { peak }}$ can be expected after short-term endurance training with SD of approximately $\pm 7 \%$, giving the estimated need for at least 14 subjects for both intervention and the control group. The subjects were randomized into a training group $(n=48)$ and a control group $(n=14$, seven men and seven women). During the study, four subjects dropped out because of personal or health related problems. Finally, 44 subjects ( 22 men and 22 women) performed the endurance training program. The Ethical Committee of the Northern Ostrobothnia Hospital District, Oulu, Finland, approved the protocol, and all the subjects gave written informed consent and the investigation conforms with the principles outlined in the Declaration of Helsinki.

\section{EXPERIMENTAL DESIGN}

On their first laboratory day, the subjects completed a health status questionnaire (simple prescreening for prior condition, e.g., medications), self-rated physical condition, self-rated mental stress, and job strain score questionnaire (Appendix; Subjects were using whole numbers as seen in Figure 1), gave written informed consent, and were assessed for BMI. All the questionnaires were completed at the quiet laboratory room. Resting electrocardiogram (ECG) was recorded to confirm their cardiac health status. On the second day, blood pressure was recorded and maximal exercise testing was performed. Use of alcohol or strenuous physical activity was not allowed during the test and on the two preceding days.

After the initial assessment, 44 subjects of the exercise group underwent highly controlled endurance training in our laboratory, whereas the control group was asked to maintain their previous level of physical activity. The training period was 2 weeks, including five consecutive sessions per week (Monday-Friday). The short intervention period was chosen on the basis of the previous studies, which shows that a well-controlled, short-term training intervention increased significantly $V_{\mathrm{O}_{2} \text { peak }}$ in sedentary subjects (Hickson et al., 1981; Mier et al., 1997; Lee et al., 2003; Hautala et al., 2006). At the end of the training intervention, all measures were repeated $48 \mathrm{~h}$ after the last training session.

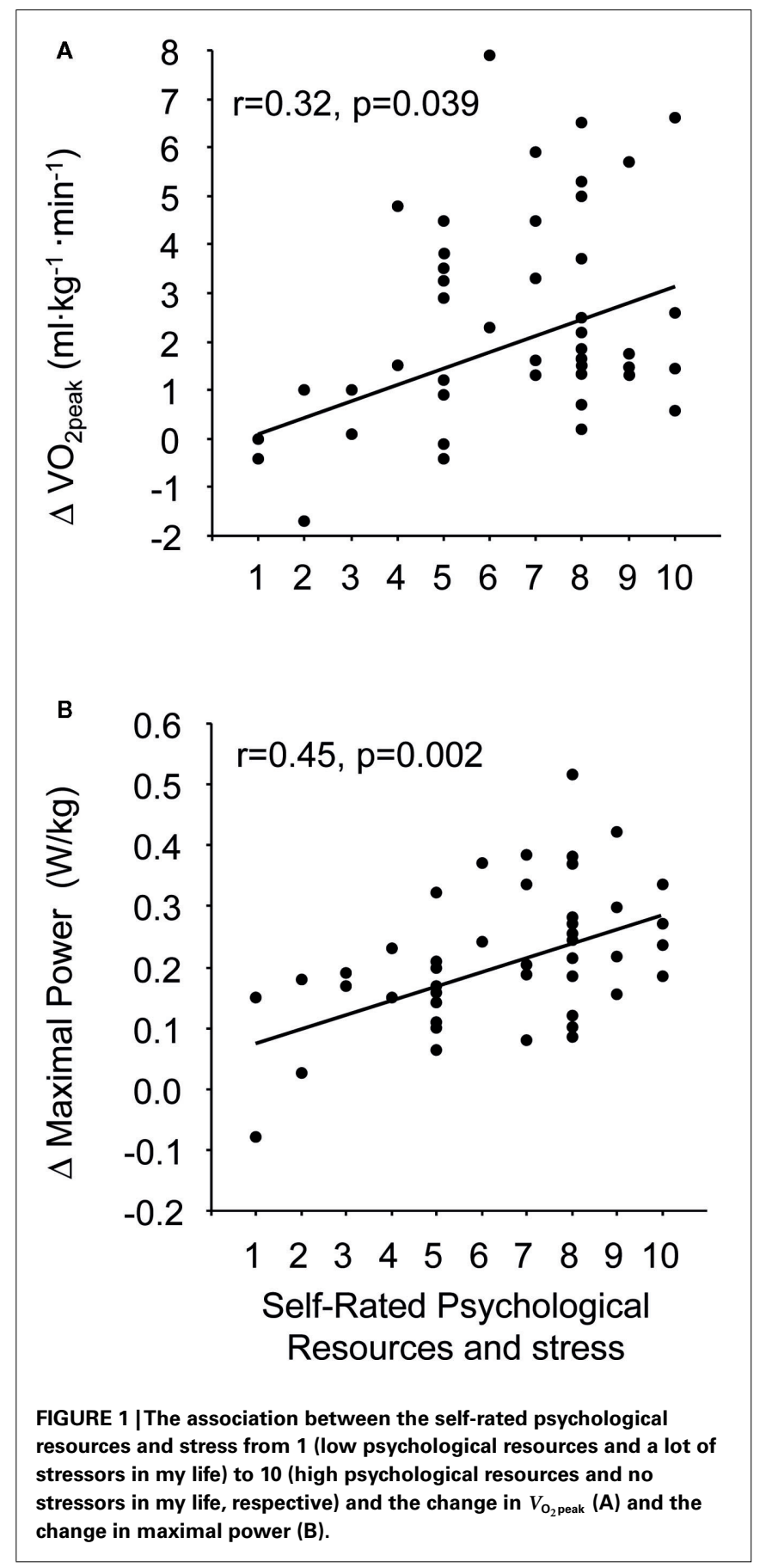

\section{ASSESSMENT OF PEAK OXYGEN CONSUMPTION}

The subjects performed a graded maximal exercise test on an 839E Monark cycle ergometer (Stockholm, Sweden). The test was started at $25 \mathrm{~W}$, and work rate was increased by $25 \mathrm{~W}$ every $2 \mathrm{~min}$ until exhaustion. Pedaling was started at $50 \mathrm{rpm}$ and increased by $5 \mathrm{rpm}$ up to $90 \mathrm{rpm}$, to achieve maximal effort. The subject were encouraged to continue cycling until they could no longer maintain the required pace, at which time the test was terminated. Ventilation (VE), gas exchange (M909 Ergospirometer, Medikro, Kuopio, Finland) and ECG (Cardiolife TEC-7721K, Nihon Kohden, 
Tokyo, Japan) were monitored continuously during the protocol. The highest oxygen uptake value measured during the test (1-min collection) was taken as the $V_{\mathrm{O}_{2} \text { peak }}$. All the subjects fulfilled the criteria for $V_{\mathrm{O}_{2} \text { peak }}$ given in the literature (i.e., respiratory exchange ratio $>1.1$ or maximal HR within \pm 10 beats of the ageappropriate reference value; Howley et al., 1995). The maximal power was assessed as average maximal watts during the last $1 \mathrm{~min}$ (absolute power and relative power, watts, and watts/body weight, respectively).

\section{EXERCISE TRAINING}

The endurance training program (Hautala et al., 2006) consisted of cycling on an 818E Monark cycle ergometer for 40 min (Stockholm, Sweden). Each exercise session consisted of a 5-min warmup period (cycling at 50 and $75 \mathrm{~W}$ resistance for women and men, respectively), followed by $30 \mathrm{~min}$ of cycling at a resistance that elicited a $\mathrm{HR}$ of $75 \%$ of maximal $\mathrm{HR}$, and ended with a 5-min cool-down period (cycling at 50 and $75 \mathrm{~W}$ resistances for women and men, respectively). Exercise intensity was closely monitored using a Polar Electro HR monitor Al and the mean HR of each training session was recorded. Endurance exercise training was planned on the basis of the recommendations of American College of Sports Medicine (ACSM, 1998). A professional instructor supervised each training session. At the end of each exercise session the subjects were asked to give perceived exertion score (RPE) on the Borg scale of 6-20 (Borg, 1973).

\section{STATISTICAL METHODS}

The results are expressed as mean $\pm \mathrm{SD}$. The normal Gaussian distribution of the data was verified by the Kolmogorov-Smirnov goodness-of-fit test. The differences within the groups were analyzed by two-factor ANOVA for repeated measures with time and interventions. Spearman's correlation analysis was performed between the self-rated questionnaires, training response, age, and baseline fitness (maximal power and $V_{\mathrm{O}_{2} \text { peak }}$, both in relative and absolute units). The data were analyzed using the SPSS software
(SPSS 12.0, SPSS inc., Chicago, USA). $P<0.05$ was considered statistically significant.

\section{RESULTS}

\section{EFFECTS OF TRAINING INTERVENTION}

The $V_{\mathrm{O}_{2} \text { peak }}$ increased $7.4 \pm 7.3 \%$ ( $p<0.001$, range from -5 to $+26 \%$ ) in exercise training group and $-0.4 \pm 7.0 \%$ (range from -16 to $+12 \% p=0.87$ ) in control group (Table 1). The maximal power increased $9.0 \pm 5.3 \%(p<0.001$, range from -4 to $+19 \%)$ in exercise training group and did not change in control group $(p=0.77$; Table 1$)$.

The average HR during all training sessions was $132 \pm 8 \mathrm{bpm}$ and was $74 \pm 2 \%$ of maximal HR. The average value of RPE at the end of exercise was $14 \pm 1$.

\section{CORRELATION BETWEEN MENTAL STRESS AND TRAINING RESPONSE}

The self-rated psychological resources/stress at the baseline condition was significantly correlated with the change in $V_{\mathrm{O}_{2} \text { peak }}$ in absolute units $\left(\mathrm{ml} \mathrm{kg}^{-1} \mathrm{~min}^{-1}, r=0.32, p=0.039\right)$ and with the changes in maximal power in absolute units $(\mathrm{W} / \mathrm{kg}, r=0.45$, $p=0.002$; Figures 1A,B). The corresponding correlations in relative units $(\%)$ were $r=0.32(p=0.033)$ and $r=0.41(p=0.006)$ for $V_{\mathrm{O}_{2} \text { peak }}$ and maximal power, respectively. The self-rated psychological resources/stress did not correlate with any fitness parameters at the baseline condition, e.g., for $V_{\mathrm{O}_{2} \text { peak }} r=-0.045$, $p=0.772$. Other self-rated questionnaires did not correlated with any variables.

The changes in fitness (expressed neither in absolute nor in\% units) did not correlate with age or baseline fitness, e.g., the correlation between $\Delta V_{\mathrm{O}_{2} \text { peak }}\left(\mathrm{ml} \mathrm{kg}^{-1} \mathrm{~min}^{-1}\right)$ and age was $r=0.11, p=0.47$ and between $\Delta V_{\mathrm{O}_{2} \text { peak }}$ and baseline $V_{\mathrm{O}_{2} \text { peak }}$ $\left(\mathrm{ml} \mathrm{kg}^{-1} \min ^{-1}\right)$ was $r=-0.039, p=0.80$.

\section{DISCUSSION}

The novel finding of the present study was the association between the self-rated mental stress and training responses after highly

Table 1 | The effects of training.

\begin{tabular}{|c|c|c|c|c|c|}
\hline & \multicolumn{2}{|c|}{ Training $(n=44)$} & \multicolumn{2}{|c|}{ Control $(n=14)$} & \multirow{2}{*}{$\begin{array}{l}\text { Group x time interaction } \\
p \text {-Value }\end{array}$} \\
\hline & Pre & Post & Pre & Post & \\
\hline Female/male & $22 / 22$ & & $7 / 7$ & & \\
\hline Age years & $41 \pm 5$ & & $42 \pm 4$ & & $p=0.58$ \\
\hline Height cm & $172 \pm 9$ & & $172 \pm 8$ & & $p=0.97$ \\
\hline Weight kg & $74 \pm 15$ & $74 \pm 14$ & $75 \pm 9$ & $75 \pm 9$ & $p=0.57$ \\
\hline $\mathrm{BMI}$ & $25 \pm 3$ & $25 \pm 3$ & $25 \pm 2$ & $24 \pm 2$ & $p=0.62$ \\
\hline BP-sys mmHg & $124 \pm 12$ & $121 \pm 11$ & $126 \pm 12$ & $124 \pm 9$ & $p=0.61$ \\
\hline BP-dia mmHg & $79 \pm 8$ & $77 \pm 7$ & $76 \pm 7$ & $77 \pm 6$ & $p=0.43$ \\
\hline \multicolumn{6}{|l|}{$V_{\mathrm{O}_{2} \text { peak }}$} \\
\hline $\mathrm{ml} \mathrm{kg}^{-1} \min ^{-1}$ & $34 \pm 7$ & $37 \pm 7$ & $34 \pm 7$ & $34 \pm 7$ & $p<0.001$ \\
\hline $\mathrm{W}_{\max } / \mathrm{kg}$ & $2.49 \pm 0.43$ & $2.71 \pm 0.43$ & $2.47 \pm 0.20$ & $2.46 \pm 0.33$ & $p<0.001$ \\
\hline $\mathrm{HR}_{\text {rest }} \mathrm{bpm}$ & $59 \pm 7$ & $57 \pm 6$ & $57 \pm 10$ & $57 \pm 10$ & $p=0.022$ \\
\hline $\mathrm{HR}_{\max } \mathrm{bpm}$ & $181 \pm 10$ & $180 \pm 10$ & $180 \pm 10$ & $179 \pm 11$ & $p=0.12$ \\
\hline $\mathrm{RER}_{\max }$ & $1.25 \pm 0.07$ & $1.25 \pm 0.07$ & $1.24 \pm 0.07$ & $1.26 \pm 0.08$ & $p=0.08$ \\
\hline
\end{tabular}

Values are mean $\pm S D$. 
controlled aerobic training intervention in healthy sedentary females and males. This data suggest that, among healthy sedentary individuals, low psychological resources and a lot of stressors at the beginning of training intervention results in low training responses in cardiorespiratory fitness and maximal power performance. Because the heterogeneity of individual training responses is not well understood, the present results provide important information of the psychological aspects of training responsiveness.

\section{EFFECTS OF TRAINING ON WELL-BEING AND MENTAL HEALTH}

A large body of cross sectional and longitudinal studies shows that physical training improves various aspects of mental health (Dunn et al., 2001; Atlantis et al., 2004; Bowen et al., 2006; Galper et al., 2006; Sjogren et al., 2006; Tolmunen et al., 2006). However, we were interested in the effects of psychological resources and stress on the training responses. A wide heterogeneity in the training responses at the individual level has been observed even after highly controlled training interventions. However, the association between a baseline mental stress and training responses is unknown. Indeed, the present study results in a clear correlation between self-rated psychological resources and stress analyzed at the baseline condition and training response after highly controlled training intervention. To the best of our knowledge there are no studies that show how psychological resources/stress or subclinical depression affects training response.

\section{INDIVIDUAL RESPONSES TO AEROBIC TRAINING}

In accordance with earlier studies, the present study also established a clear variation in the training response after controlled aerobic training intervention. The HERITAGE Family Study based on 720 healthy sedentary subjects showed that age, sex, race, and baseline fitness level accounted together only for $11 \%$ of the variance in the adaptation to aerobic training. The strongest predictors of the training response were the sex and the age of the subject's in this order (Bouchard and Rankinen, 2001). In the present study after highly controlled short-term training intervention age or baseline aerobic fitness were not associated with training response. Our population is rather small and the age range as well as aerobic fitness range are very narrow and may well explain the lack of association between age, baseline fitness and training response.

\section{POTENTIAL MECHANISMS FOR LOW TRAINING RESPONSE}

The mechanisms underlying the relationship between the baseline self-rated psychological resources/stress and the training response remain speculative. Stress state may effects to adaptation to training so that training response remains low. In other words, the level of stress is already too high for subjects in low psychological resources group to handle more external stimuli, e.g., exercise training (Huang et al., 2006).

In the LiVicordia Study good self-rated health $(\mathrm{SRH})$ is related to psychosocial resources and strong cortisol respond to acute stress (Kristenson et al., 2005). In the LiVicordia Study men with good SRH, compared to men with poor SRH had lower baseline levels of cortisol in saliva samples and so they have better ability to adapt to stress. Lower cortisol response to acute stress has been reported in subjects with chronic stress conditions (Benschop et al., 1994; Pruessner et al., 1999). In the present study low psychological resources and a lot of stressors in life were associated to low training respond. If the self-rated psychological resources/stress reflect also the cortisol level or vital exhaustion state like SRH does, it may also offer one possible explanation to low training respond. Subjects with low psychological resources and a lot of stressors in life may have attenuated cortisol response to acute stress resulting in a low training response in cardiovascular fitness. The association between the level of salivary DHEA-S and training adaptation also supports that concept (Huang et al., 2006).

\section{LIMITATIONS OF THE STUDY}

The main limitation of this study is the method of the mental stress assessment, which is quite narrow and our questionnaire is not published elsewhere. Furthermore, the validity and reliability of the tool has not been studied. On the other hand it was able to show that the self-rated psychological resources combined with the self-rated number of stressors in daily life at the baseline condition correlated with the change in fitness after training intervention. One cannot be certain what exactly the question "How would you assess your psychological resources at the moment?" reflects. However, the question is aiming to measure also stress state as it continuous; "I have a plenty, some or no stressors in my life." Secondly, in many studies individuals experience about their health has been estimated by the question "How would you assess your own health?" and this SRH has been clearly showed its value in research. For example SRH is a strong predictor of mortality (Idler and Benyamini, 1997). The second limitation of this study was that we did not measure self-rated psychological resources/stress at the end of the training intervention because we did not think that the evaluation on self-rated resources/stress could change in such a short time.

\section{CONCLUSION}

The present study provides novel information on the contribution of the psychological aspects to the training response. The changes in fitness were very low or absent in the subjects who experience their psychological resources low and a lot of stressors in their daily life at the beginning of short-term training intervention. These observations may have some practical implications. The measurement of mental stress or/and self-rated mental health may become an important variable in the analysis of individual training responses after exercise interventions. Also mental stress should be noticed in the exercise training counseling for the different individuals particularly in cardiac patients which are known to suffer various mood disorders. However, the present findings are very preliminary due to the small sample size and very limited tools to measure mental stress.

\section{ACKNOWLEDGMENTS}

This research was funded by grants from the Ministry of Education (Helsinki, Finland) and Finnish Funding Agency for Technology and Innovation, TEKES (Helsinki, Finland). The authors appreciate the technical and financial support received from Polar Electro (Kempele, Finland). The authors have no conflict of interests to report. The results of the present study do not constitute endorsement by ACSM. 


\section{REFERENCES}

ACSM. (1998). American College of Sports Medicine Position Stand. The recommended quantity and quality of exercise for developing and maintaining cardiorespiratory and muscular fitness, and flexibility in healthy adults. Med. Sci. Sports Exerc. 30, 975-991.

Atlantis, E., Chow, C. M., Kirby, A., and Singh, M. F. (2004). An effective exercise-based intervention for improving mental health and quality of life measures: a randomized controlled trial. Prev. Med. 39, 424-434.

Benschop, R. J., Brosschot, J. F., Godaert, G. L., De Smet, M. B., Geenen, R., Olff, M., Heijnen, C. J., and Ballieux, R. E. (1994). Chronic stress affects immunologic but not cardiovascular responsiveness to acute psychological stress in humans. Am. J. Physiol. 266, R75-R80.

Billman, G. E. (2002). Aerobic exercise conditioning: a nonpharmacological antiarrhythmic intervention. J. Appl. Physiol. 92, 446-454.

Borg, G. A. (1973). Perceived exertion: a note on "history" and methods. Med. Sci. Sports 5, 90-93.

Bouchard, C. (1995). Individual differences in the response to regular exercise. Int. J. Obes. Relat. Metab. Disord. 19(Suppl. 4), S5-S8.

Bouchard, C., An, P., Rice, T., Skinner, J. S., Wilmore, J. H., Gagnon, J., Perusse, L., Leon, A. S., and Rao, D. C. (1999). Familial aggregation of $\mathrm{VO}(2 \mathrm{max})$ response to exercise training: results from the HERITAGE Family Study. J. Appl. Physiol. 87, 1003-1008.

Bouchard, C., and Rankinen, T. (2001). Individual differences in response to regular physical activity. Med. Sci. Sports Exerc. 33, S446-S451; discussion S452-S453.

Bouchard, C., Sarzynski, M. A., Rice, T. K., Kraus, W. E., Church, T. S., Sung, Y. J., Rao, D. C., and Rankinen, T. (2011). Genomic predictors of the maximal $\mathrm{O}$ uptake response to standardized exercise training programs. J. Appl. Physiol. 110, 1160-1170.

Bowen, D. J., Fesinmeyer, M. D., Yasui, Y., Tworoger, S., Ulrich, C. M., Irwin, M. L., Rudolph, R. E., Lacroix, K. L., Schwartz, R. R., and Mctiernan, A. (2006). Randomized trial of exercise in sedentary middle aged women: effects on quality of life. Int. J. Behav. Nutr. Phys. Act. 3, 34.

Cruess, D. G., Antoni, M. H., Kumar, M., Ironson, G., Mccabe, P., Fernandez, J. B., Fletcher, M., and Schneiderman, N. (1999). Cognitive-behavioral stress management buffers decreases in dehydroepiandrosterone sulfate (DHEA-S) and increases in the cortisol/DHEA-S ratio and reduces mood disturbance and perceived stress among HIV-seropositive men. Psychoneuroendocrinology 24, 537-549.

Dunn, A. L., Trivedi, M. H., and O'Neal, H. A. (2001). Physical activity doseresponse effects on outcomes of depression and anxiety. Med. Sci. Sports Exerc. 33, S587-S597; discussion 609-610.

Galper, D. I., Trivedi, M. H., Barlow, C. E., Dunn, A. L., and Kampert, J. B. (2006). Inverse association between physical inactivity and mental health in men and women. Med. Sci. Sports Exerc. 38, 173-178.

Hagberg, J. M., Rankinen, T., Loos, R. J., Perusse, L., Roth, S. M., Wolfarth, B., and Bouchard, C. (2011). Advances in exercise, fitness, and performance genomics in 2010. Med. Sci. Sports Exerc. 43, 743-752.

Hautala, A. J., Kiviniemi, A. M., Makikallio, T. H., Kinnunen, H., Nissila, S., Huikuri, H. V., and Tulppo, M. P. (2006). Individual differences in the responses to endurance and resistance training. Eur. J. Appl. Physiol. 96, 535-542.

Hautala, A. J., Leon, A. S., Skinner, J. S., Rao, D. C., Bouchard, C., and Rankinen, T. (2007). Peroxisome proliferator-activated receptor-delta polymorphisms are associated with physical performance and plasma lipids: the HERITAGE Family Study. Am. J. Physiol. Heart Circ. Physiol. 292, H2498-H2505.

Hautala, A. J., Makikallio, T. H., Kiviniemi, A., Laukkanen, R. T., Nissila, S., Huikuri, H. V., and Tulppo, M. P. (2003). Cardiovascular autonomic function correlates with the response to aerobic training in healthy sedentary subjects. Am. J. Physiol. Heart Circ. Physiol. 285, H1747-H1752.

Herbert, J. (1998). Neurosteroids, brain damage, and mental illness. Exp. Gerontol. 33, 713-727.

Hickson, R. C., Hagberg, J. M., Ehsani, A. A., and Holloszy, J. O. (1981). Time course of the adaptive responses of aerobic power and heart rate to training. Med. Sci. Sports Exerc. 13, 17-20.

Howley, E. T., Bassett, D. R. Jr., and Welch, H. G. (1995). Criteria for maximal oxygen uptake: review and commentary. Med. Sci. Sports Exerc. 27, 1292-1301.

Huang, Y. J., Chen, M. T., Fang, C. L., Lee, W. C., Yang, S. C., and
Kuo, C. H. (2006). A possible link between exercise-training adaptation and dehydroepiandrosterone sulfate- an oldest-old female study. Int. J. Med. Sci. 3, 141-147.

Idler, E. L., and Benyamini, Y. (1997). Self-rated health and mortality: a review of twenty-seven community studies. J. Health Soc. Behav. 38, 21-37.

Kesäniemi, Y. K., Danforth, E. Jr., Jensen, M. D., Kopelman, P. G., Lefebvre, P., and Reeder, B. A. (2001). Dose-response issues concerning physical activity and health: an evidence-based symposium. Med. Sci. Sports Exerc. 33, S351-S358.

Kristenson, M., Olsson, A. G., and Kucinskiene, Z. (2005). Good selfrated health is related to psychosocial resources and a strong cortisol response to acute stress: the LiVicordia study of middle-aged men. Int. J. Behav. Med. 12, 153-160.

Kurl, S., Laukkanen, J. A., Rauramaa, R., Lakka, T. A., Sivenius, J., and Salonen, J. T. (2003). Cardiorespiratory fitness and the risk for stroke in men. Arch. Intern. Med. 163, 1682-1688.

LaMonte, M. J., Barlow, C. E., Jurca, R., Kampert, J. B., Church, T. S. and Blair, S. N. (2005). Cardiorespiratory fitness is inversely associated with the incidence of metabolic syndrome: a prospective study of men and women. Circulation 112, 505-512.

Laukkanen, J. A., Kurl, S., Salonen, R., Rauramaa, R., and Salonen, J. T. (2004). The predictive value of cardiorespiratory fitness for cardiovascular events in men with various risk profiles: a prospective populationbased cohort study. Eur. Heart J. 25, 1428-1437.

Lee, C. M., Wood, R. H., and Welsch, M. A. (2003). Influence of shortterm endurance exercise training on heart rate variability. Med. Sci. Sports Exerc. 35, 961-969.

Lee, W. C., Chen, S. M., Wu, M. C. Hou, C. W., Lai, Y. C., Laio, Y. H., Lin, C. H., and Kuo, C. H. (2006). The role of dehydroepiandrosterone levels on physiologic acclimatization to chronic mountaineering activity. High Alt. Med. Biol. 7, 228-236.

Mier, C. M., Turner, M. J., Ehsani, A. A., and Spina, R. J. (1997). Cardiovascular adaptations to 10 days of cycle exercise. J. Appl. Physiol. 83, 1900-1906.

Myers, J., Prakash, M., Froelicher, V., Do, D., Partington, S., and Atwood,
J. E. (2002). Exercise capacity and mortality among men referred for exercise testing. N. Engl. J. Med. 346, 793-801.

Pruessner, J. C., Hellhammer, D. H., and Kirschbaum, C. (1999). Burnout, perceived stress, and cortisol responses to awakening. Psychosom. Med. 61, 197-204.

Regelson, W., Loria, R., and Kalimi, M. (1988). Hormonal intervention: "buffer hormones" or "state dependency". The role of dehydroepiandrosterone (DHEA), thyroid hormone, estrogen and hypophysectomy in aging. Ann. N. Y. Acad. Sci. 521, 260-273.

Sjogren, T., Nissinen, K. J., Jarvenpaa, S. K., Ojanen, M. T., Vanharanta, H., and Malkia, E. A (2006). Effects of a physical exercise intervention on subjective physical well-being, psychosocial functioning and general well-being among office workers: a cluster randomized-controlled cross-over design. Scand. J. Med. Sci. Sports 16, 381-390.

Timmons, J. A., Knudsen, S., Rankinen, T., Koch, L. G., Sarzynski, M., Jensen, T., Keller, P., Scheele, C., Vollaard, N. B., Nielsen, S., Akerstrom, T., Macdougald, O. A., Jansson, E., Greenhaff, P. L., Tarnopolsky, M. A., Van Loon, L. J., Pedersen, B. K., Sundberg, C. J., Wahlestedt, C., Britton, S. L., and Bouchard, C. (2010). Using molecular classification to predict gains in maximal aerobic capacity following endurance exercise training in humans. J. Appl. Physiol. 108, 1487-1496.

Tolmunen, T., Laukkanen, J. A., Hintikka, J., Kurl, S., Viinamaki, H., Salonen, R., Kauhanen, J., Kaplan, G. A., and Salonen, J. T. (2006). Low maximal oxygen uptake is associated with elevated depressive symptoms in middleaged men. Eur. J. Epidemiol. 21, 701-706.

Tulppo, M. P., Hautala, A. J., Makikallio, T. H., Laukkanen, R. T., Nissila, S., Hughson, R. L., and Huikuri, H. V. (2003). Effects of aerobic training on heart rate dynamics in sedentary subjects. J. Appl. Physiol. 95, 364-372.

Tulppo, M. P., Kiviniemi, A. M., Hautala, A. J., Kallio, M., Seppanen, T., Makikallio, T. H., and Huikuri, H. V. (2005). Physiological background of the loss of fractal heart rate dynamics. Circulation 112 , 314-319.

Wang, H. T., Chen, S. M., Lee, S. D., Hsu, M. C., Chen, K. N., Liou, Y. 
F., and Kuo, C. H. (2009). The role of DHEA-S in the mood adjustment against negative competition outcome in golfers. J. Sports Sci. 27, 291-297.

Conflict of Interest Statement: The authors declare that the research was conducted in the absence of any commercial or financial relationships that could be construed as a potential conflict of interest.

Received: 12 October 2011; accepted: 26 February 2012; published online: 12 March 2012.
Citation: Ruuska PS, Hautala AJ, Kiviniemi AM, Mäkikallio TH and Tulppo MP (2012) Self-rated mental stress and exercise training response in healthy subjects. Front. Physio. 3:51. doi: 10.3389/fphys.2012.00051

This article was submitted to Frontiers in Clinical and Translational Physiology, a specialty of Frontiers in Physiology.
Copyright (c) 2012 Ruuska, Hautala, Kiviniemi, Mäkikallio and Tulppo. This is an open-access article distributed under the terms of the Creative Commons Attribution Non Commercial License, which permits non-commercial use, distribution, and reproduction in other forums, provided the original authors and source are credited. 


\section{APPENDIX}

Dear participant,

Name:

We request you to assess your physical condition, your psychological resources/stress and your job strain. Mark the point of the line that corresponds to those best. You can also specify your answer to the space under the line.

\section{How would you describe your physical condition at the moment?}

$1=$ my condition is poor, I get tired very easily

$5=$ my condition is mediocre, I can take some physical strain

$10=\mathrm{I}$ am in great condition and I can take plenty of physical strain

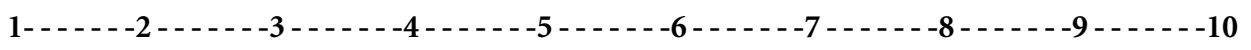

2. How would you assess your psychological resources and stress at the moment? Consider you entire life situation, in other words both work and personal life.

$1=$ psychological resources are very low, there is plenty of stressors in my life

$5=$ psychological resources are moderate, there is some stressors in my life

$10=$ psychological resources are good, stressors in my life are not worth mentioning

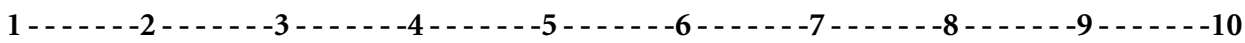

3. How much your work is wearing your psychological resources?

$1=$ work does not wear my psychological resources at all

$5=$ work wears a lot my psychological resources, but I still have energy on free -time

$10=$ work is consuming all my psychological resources, I do not have the energy to do anything else

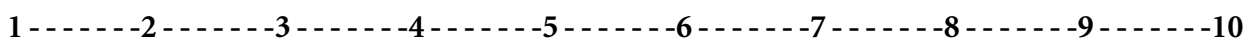

4. What kind of goals and hopes you have considering your well -being and this study?

Please, write your goals and hopes in order of importance. 